\title{
Surface Instability in Windblown Sand
}

\author{
Douglas A. Kurtze \\ Department of Physics, North Dakota State University, Fargo, ND 58105-5566, USA \\ Joseph A. Both and Daniel C. Hong \\ Department of Physics, Lewis Laboratory, Lehigh University, Bethlehem, PA 18015, USA
}

\begin{abstract}
We investigate the formation of ripples on the surface of windblown sand based on the one-dimensional model of Nishimori and Ouchi [Phys. Rev. Lett. 71, 197 (1993)], which contains the processes of saltation and grain relaxation. We carry out a nonlinear analysis to determine the propagation speed of the restabilized ripple patterns, and the amplitudes and phases of their first, second, and third harmonics. The agreement between the theory and our numerical simulations is excellent near the onset of the instability. We also determine the Eckhaus boundary, outside which the steady ripple patterns are unstable.
\end{abstract}

PACS numbers: 47.54.+r, 45.70-n, 92.10.Wa

\section{Introduction}

Since the pioneering work of Bagnold [1], many researchers have investigated the complex dynamics of dry granular materials at a surface [2-6]. Dry granular materials are assemblies of macroscopic objects that interact with each other essentially via a hard core repulsive potential. Hence they are loosely connected, particularly at the surface. When those grains at the surface are exposed to a wind, they can readily be ejected and carried by the wind until gravity eventually pulls them back to the surface. The dynamics of a single grain is rather simple, given by the Newtonian trajectory of a point particle. Even so, experiments have shown that the collective response of the grains can become exceedingly complex, ranging from formation of simple ripple patterns to ridges and dunes to violent tornadoes [2]. Our current understanding of such complex phenomena remains mostly confined to compiling 
data on experimental observations. With regard to the formation of ripple patterns, however, there have been some attempts to construct a simple yet physical continuum model.

We will investigate the continuum model due to Nishimori and Ouchi [3]. The Nishimori-Ouchi (NO) model of ripple patterns accounts for two elementary processes of sand transportation by the wind which have been identified by investigators in aeolian sand dynamics, namely saltation and creep $[1,2]$. Saltation refers to the process by which surface grains are ejected into the air under the influence of a strong wind, and are blown downwind where they collide with other surface grains. There they transfer momentum to these downwind grains, which may themselves be ejected in turn, thereby continuing the process (see Fig. 1). Creep is the surface movement of grains too heavy to be ejected into the air but light enough to be pushed along the surface. Creep also describes the surface movement of grains on hills under the influence of gravity. Previous studies based on the NO model have been confined largely to linear stability analysis and Monte Carlo simulations of a lattice version with simple rules for the grain dynamics [3,11]. The purpose of this paper is to go one step farther by carrying out a nonlinear analysis of the continuum model and uncovering some of the features of ripple formation that are inaccessible to linear analysis.

In particular, we carry out a weakly nonlinear analysis valid near the onset of instability of a flat sandbed to determine the amplitude, shape, and propagation speed of the ripple pattern that forms in this regime. We also compare these results with our numerical integrations of the model equations. These computations are rather unusual because the model lacks an up-down symmetry, and especially because accounting for saltation makes the model nonlocal in space. We find, however, that the process of pattern selection in this simple one-dimensional system, in particular the selection of the wavelength and speed of the patterns [7], is similar to what is seen in more complicated multidimensional systems such as directional solidification [8] or directional viscous fingering [9]. That is, the wavelength of the final pattern depends on the initial conditions, and may lie anywhere within a band of linearly stable final states. The stable band turns out to be somewhat wider than in most other models.

In the next section we review the Nishimori-Ouchi model equations [3], point out a physical symmetry which they violate, and propose a simple modification of the model which respects that symmetry. In Section III we 
carry out a linear stability analysis of the flat-sandbed solution of the model, both for the original Nishimori-Ouchi equations and for our modification. We extend this in Section IV to give a weakly nonlinear analysis for both forms of the model. Section V presents our numerical calculations and compares them with the results of the weakly nonlinear analysis. The results are discussed in the final section.

\section{One-Dimensional Model for Windblown Sand}

The starting point of the Nishimori-Ouchi (NO) model [3] is a local conservation law for sand grains. Let $h(x, t)$ be the local height of the sand bed at position $x$ and time $t$, measured from some reference level. The height increases when grains are added at position $x$. We write

$$
\frac{\partial h}{\partial t}+\frac{\partial J_{l}}{\partial x}=Q_{n l},
$$

where $J_{l}(x, t)$ is a local flux of grains in the positive direction at $x$, and $Q_{n l}(x, t)$ is the net input of grains at $x$ due to nonlocal processes.

The expression for $J_{l}$ embodies a model of creep. Nishimori and Ouchi choose $J_{l}=-D(\partial h / \partial x)$. Note that this merely expresses the tendency of grains to roll downhill; it does not include any bias favoring motion in the direction of the wind.

Saltation is modeled by gain and loss terms in the nonlocal transfer rate $Q_{n l}$. Let $N(x, t)$ denote the outward saltation flux of particles from $x$ at time $t$. That is, let $N(x, t) d x$ be the number of particles per unit time taking off from positions between $x$ and $x+d x$. The loss term in $Q_{n l}$ is then $-A N(x, t)$, where $A$ is a scale parameter. The gain term is proportional to the rate at which particles arrive at $x$ from other locations $\xi$ upwind of $x$. Suppose all particles which take off from the interval $(\xi, \xi+d \xi)$ subsequently land in the interval $(x, x+d x)$. Then the number of particles per unit time landing in this latter interval of length $d x$ is $N(\xi, t) d \xi$, so the gain term in the saltation flux is then $A N(\xi, t)(d \xi / d x)$. It is possible to have more than one $\xi$ which satisfies this equation for a given $x$. That is, grains landing at $x$ may have come from more than one takeoff point $\xi$. If this is the case, then the input term in the evolution equation should be summed over the different values of $\xi$. 
Note that evaluating $N(\xi)$ at time $t$ neglects the flight time of the incoming grains; we expect the evolution of the sandbed profile to take place on a much longer time scale than this, so that the time delay between takeoff and landing should be unimportant. Indeed, experiments on ripple formation by sand transported by water [10] show the evolution of the ripple pattern occurring on time scales of several hours.

Combining the various contributions to the flux and substituting into the general conservation law for $h$ gives the model evolution equation for the sandbed profile,

$$
\frac{\partial h}{\partial t}=\frac{\partial}{\partial x} D \frac{\partial h}{\partial x}+A\left[N(\xi, t) \frac{d \xi}{d x}-N(x, t)\right] .
$$

Note that this equation is nonlocal in $x$, as a result of the saltation gain term, which depends on conditions at a position $\xi$ which is a finite distance upwind of $x$.

To complete the model, we must now specify the saltation function, an equation for the flight length of a single grain. In general, the locations $x$ and $\xi$ in the evolution equation will be related by $x=\xi+L$, where $L$ is the horizontal length an ejected grain travels from takeoff to landing. This will depend on the size of the grain, its speed when it takes off, the wind velocity profile, and the topography of the sandbed itself. Nishimori and Ouchi proposed the simple ansatz

$$
L=L_{0}+b h(\xi, t)
$$

Here, $L_{0}$ is a parameter proportional to the shear stress of the wind at the surface, or more precisely to the friction velocity of the wind on the sand surface [11], and $b$ in general depends on the average drag force on the grain. Nishimori and Ouchi took both $L_{0}$ and $b$ to be constant, essentially assuming the wind velocity to be a constant, independent of $x$ and $t$ and unaffected by changes in the sandbed profile.

Equation (3) merely indicates that the higher the takeoff point of a grain in saltation, the longer its trajectory. As Nishimori and Ouchi point out [3], this amounts to assuming that the height and topography at the point of landing may be neglected, and that only the surface height (as opposed to local topography) is important at the takeoff point. While this may be reasonable if $h(x)$ is everywhere close to zero, it does violate a symmetry 
of the physical problem, namely that the dynamics should be unaffected if we add any constant to $h$, thus changing our reference level. To restore this symmetry, it may be more appropriate to take the saltation function to be

$$
L=L_{0}+b[h(\xi)-h(x)]
$$

where $\xi$ is the takeoff point and $x$ is the landing point. We will discuss the effects of this modification below.

For convenience, we now put the model into dimensionless form. Taking $L_{0}, b$ and $D$ to be constants, we choose $L_{0}$ to be the unit of horizontal length, $L_{0} / b$ to be the unit of vertical length (i.e., of $h$ ), and $L_{0}^{2} / D$ to be the time unit. Further, we define $J(x, t)=\left(A b L_{0} / D\right) N(x, t)$, a dimensionless measure of the outward grain flux due to saltation. With these definitions, the evolution equation (2) becomes

$$
\frac{\partial h}{\partial t}=\frac{\partial^{2} h}{\partial x^{2}}+J(\xi, t) \frac{d \xi}{d x}-J(x, t),
$$

with the original $\mathrm{NO}$ saltation relation becoming the condition

$$
x=\xi+1+h(\xi, t) .
$$

The model simplifies further if we choose $J(x, t)$ to be a constant $J$ independent of $x$ and $t$, an assumption whose physical content is that the wind is uniform and there is no flux dependence on surface height. The evolution equation is then

$$
\frac{\partial h}{\partial t}=\frac{\partial^{2} h}{\partial x^{2}}+J\left(\frac{d \xi}{d x}-1\right)
$$

This is the form of the problem which we will analyze below, using both the NO saltation relation (6) and our symmetric modification of it,

$$
x=\xi+1+h(\xi, t)-h(x, t) .
$$

\section{Linear Stability Analysis}

We first note that a flat sandbed, $h=h_{0}=$ constant, is always a steadystate solution of the model, for either choice of saltation relation. For the symmetric saltation relation (8) this always gives $\xi=x-1$, while for the 
NO relation (6) we have $\xi=x-1-h_{0}$. In the latter case, however, we may then redefine the length and time units - and the value of $J$ - to map the solution with any finite $h_{0}$ (provided $h_{0}>-1$ ) onto the solution with $h_{0}=0$. Specifically, we would take the horizontal length unit to be $L_{0}\left(1+h_{0}\right)$ instead of $L_{0}$, and $J$ would then be $A b L_{0}\left(1+h_{0}\right) N / D$ rather than $A b L_{0} N / D$. Thus we will take $h=0$ to be the steady state whose stability we will investigate.

When $h$ is small, we may linearize the NO saltation relation to get

$$
\xi \approx x-1-h(x-1)
$$

so that there is a single, unique $\xi$ for each $x$. From this we obtain $d \xi / d x=$ $1-h^{\prime}(x-1)$, where the prime indicates partial differentiation with respect to $x$. The linearized evolution equation is then

$$
\frac{\partial h}{\partial t}=h^{\prime \prime}(x, t)-J h^{\prime}(x-1, t) .
$$

Linear stability analysis proceeds in the usual way: we write $h(x, t)$ as a linear combination of Fourier modes,

$$
h(x, t)=\int h_{k}(t) \exp (i k x) d k,
$$

substitute this into the linearized evolution equation, and note that - even with the nonlocal term present - the modes do not couple. Thus we find that each mode grows or decays exponentially with time,

$$
h_{k}(t) \propto \exp \left[\left(\sigma_{k}-i \omega_{k}\right) t\right]
$$

with $[3,11]$

$$
\begin{aligned}
\sigma_{k} & =-k^{2}-J k \sin k \\
\omega_{k} & =J k \cos k .
\end{aligned}
$$

If we use the symmetric saltation relation instead of the NO relation, then the linearized evolution equation has an extra term $+J h^{\prime}(x, t)$ on the right side. This leaves $\sigma_{k}$ unchanged, but replaces (14) by $\omega_{k}=-J k(1-\cos k)$.

The locus $\sigma_{k}=0$ in the $J-k$ plane defines a stability boundary. Points on one side of the boundary represent perturbations which have a positive growth rate $\sigma_{k}$, while points on the other side represent perturbations with 
negative growth rates which are therefore suppressed in the solution. Thus we expect that solutions of the full differential equation will consist only of modes whose wave numbers are on the unstable side of the boundary. The onset of instability of the flat sandbed occurs at the value $J_{c}$ of $J$ for which only a single mode, with wave number $k_{c}$, is marginally stable and no other modes are unstable. These critical values may be determined by solving $\sigma=0$ and $d \sigma / d k=0$ simultaneously, which yields

$$
\begin{aligned}
J_{c} \sin k_{c} & =-k_{c}, \\
J_{c} \cos k_{c} & =-1
\end{aligned}
$$

Eliminating $J_{c}$ gives

$$
\tan k_{c}=k_{c} \text {. }
$$

Thus the critical values are computed as $k_{c}=4.493$ and $J_{c}=4.603$. The wavelength of the marginal mode (in units of $L_{0}$ ) is $\lambda_{c}=2 \pi / k_{c}=1.398$, somewhat longer than the flight distance of a grain in saltation. For $k=k_{c}$, the NO saltation relation leads to $\omega_{c}=-k_{c}$, so the phase velocity of the marginal mode is $v=\omega_{c} / k_{c}=-1$. With the symmetric saltation relation we get $v=-\left(1+J_{c}\right)=-5.60$. This is a surprising result of the model, that while the sand grains that form the ripples are blown downwind, the ripple pattern itself drifts upwind. The group velocity, however, is large and positive: From (114) we get $d \omega_{k} / d k=J(-k \sin k+\cos k)$, which goes to $k_{c}^{2}-1=19.19$ at the critical point. For the symmetric saltation relation, the group velocity is lower by $J$, so at critical it is 14.58 . Note that all velocities are in units of $D / L_{0}$.

If we make the problem two-dimensional, allowing the sandbed to extend in both $x$ and $y$ directions, very little changes. The creep term in the evolution equation becomes $D \nabla^{2} h$, and as a result the expression for $\sigma_{k}$ changes to

$$
\sigma\left(k, k_{y}\right)=-k^{2}-k_{y}^{2}-J k \sin k,
$$

where $k$ is now the $x$ component of the wave vector of the Fourier mode and $k_{y}$ is its $y$ component. Clearly, the linear growth rate for a mode with nonzero $k_{y}$ is always less than the rate for the corresponding mode with $k_{y}=0$. Thus we do not expect to see instabilities in which the transverse shape of the ripples becomes wavy, since the first instability to occur is against a mode in which the ripples are parallel to the $y$ axis. 


\section{Nonlinear Analysis}

We now carry out a weakly nonlinear analysis to determine the amplitude, shape, and propagation velocity of the restabilized ripple patterns which form when $J$ is slightly above its critical value $J_{c}$. The nonlocality of the model, the dispersion in the imaginary part of the linear growth rate, and the lack of an up-down symmetry lead to some unusual features in the analysis.

We begin with the assumption that the fundamental wave number $k$ of the pattern which develops does not deviate much from the critical value $k_{c}$ when $J$ is near $J_{c}$. Hence we define a small parameter $\epsilon$ by setting

$$
J=J_{c}+\epsilon^{2},
$$

and then define a scaled wave number deviation $q$ by writing

$$
k=k_{c}+\epsilon q .
$$

This is the appropriate scaling for the wave number because the stability boundary is approximately quadratic in $k-k_{c}$ and linear in $J$ near its maximum. Substituting these expressions into the linear growth rate (13) and expanding to second order in $\epsilon$ gives

$$
\sigma_{k}=\frac{1}{2} \epsilon^{2} k_{c}^{2}\left(\frac{2}{J_{c}}-q^{2}\right)+O\left(\epsilon^{3}\right) .
$$

Furthermore, from the expression (14) for $\omega$ we find that the phase velocity of the ripples is given by

$$
v=\omega / k=-1+\epsilon k_{c} q-\frac{1}{2} \epsilon^{2}\left(\frac{2}{J_{c}}-q^{2}\right)+O\left(\epsilon^{3}\right) .
$$

The first stage of the nonlinear analysis consists of expanding the evolution equation (7) in powers of $h$, assuming the overall amplitude of $h$ is small. To do this, we may rewrite the NO saltation relation (6) in the form

$$
\xi=x-1-h(\xi, t),
$$

repeatedly substitute this expression for $\xi$ back into the $h(\xi, t)$ on the right side, and finally expand in powers of $h$. Differentiating the result with respect to $x$ then gives

$$
\frac{d \xi}{d x}=1-h^{\prime}(x-1, t)+\frac{1}{2}\left[h^{2}(x-1, t)\right]^{\prime \prime}-\frac{1}{6}\left[h^{3}(x-1, t)\right]^{\prime \prime \prime}+O\left(h^{4}\right) .
$$


To third order in $h$, then, the evolution equation becomes

$$
\frac{\partial h(x, t)}{\partial t}=h^{\prime \prime}(x, t)-J h^{\prime}(x-1, t)+\frac{J}{2}\left[h^{2}(x-1, t)\right]^{\prime \prime}-\frac{J}{6}\left[h^{3}(x-1, t)\right]^{\prime \prime \prime}+\cdots
$$

This is the equation whose ripple solutions we will presently compute.

It is remarkable that the expansion of $d \xi / d x$ has such an economical form. In fact it is not difficult to show that the pattern continues to all orders in $h$. To see this, consider the integral

$$
I_{f} \equiv \int_{-\infty}^{\infty} f(x-1) \frac{d \xi}{d x} d x
$$

where $\xi(x)$ is given by the saltation relation (6) and $f$ is a test function which is integrable and infinitely differentiable, but otherwise arbitrary. We now change variables in this integral from $x$ to $\xi$,

$$
I_{f}=\int_{-\infty}^{\infty} f(\xi+h(\xi)) d \xi
$$

and expand the integrand in powers of $h$ to get

$$
I_{f}=\int \sum_{k=0}^{\infty} \frac{1}{k !} \frac{d^{k} f(\xi)}{d \xi^{k}} h^{k}(\xi) d \xi .
$$

Next we integrate the $k$ th term by parts $k$ times to get

$$
I_{f}=\int f(\xi) \sum_{k=0}^{\infty} \frac{(-1)^{k}}{k !} \frac{d^{k} h^{k}(\xi)}{d \xi^{k}} d \xi,
$$

and finally change variables again from $\xi$ to $x \equiv \xi+1$,

$$
I_{f}=\int_{-\infty}^{\infty} f(x-1) \sum_{k=0}^{\infty} \frac{(-1)^{k}}{k !} \frac{d^{k} h^{k}(x-1)}{d x^{k}} d x
$$

This result has the same form as the original expression for $I_{f}$, but with $d \xi / d x$ replaced by an expansion. However, since the test function $f$ is arbitrary, this requires the expansion and $d \xi / d x$ to be equal:

$$
\frac{d \xi}{d x}=\sum_{k=0}^{\infty} \frac{(-1)^{k}}{k !} \frac{d^{k} h^{k}(x-1)}{d x^{k}} .
$$


We now turn to the second stage of the calculation, namely finding solutions to the third-order approximation (24) to the evolution equation. We assume the solution will have a fundamental wave number $k$ in the unstable range, with an amplitude of order $\epsilon$. The quadratic terms in the evolution equation will then generate a Fourier component in the solution with wave number $2 k$ and possibly a constant term, and the cubic terms will lead to a component with wave number $3 k$. Thus we write

$$
\begin{aligned}
h(x, t) \approx \epsilon M(t) \cos (k x-\phi(t)) & +\epsilon^{2} M_{0}(t)+\epsilon^{2} M_{2}(t) \cos \left[2(k x-\phi(t))+\theta_{2}(t)\right] \\
& +\epsilon^{3} M_{3}(t) \cos \left[3(k x-\phi(t))+\theta_{3}(t)\right]+\cdots,(31)
\end{aligned}
$$

allowing phase differences among the various Fourier components. We substitute this ansatz into the evolution equation and expand in powers of $\epsilon$. Then the coefficients of $\cos (k x-\phi(t))$ and $\sin (k x-\phi(t))$ give equations for the fundamental amplitude $M(t)$ and phase $\phi(t)$ :

$$
\begin{gathered}
\dot{M}=\sigma_{k} M-\epsilon^{2} J k^{2} M_{0} M \cos k-\frac{\epsilon^{2}}{2} J k^{2} M_{2} M \cos \left(k-\theta_{2}\right) \\
+\frac{\epsilon^{2}}{8} J k^{3} M^{3} \sin k+O\left(\epsilon^{4}\right) \\
\dot{\phi}=\omega_{k}-\epsilon^{2} J k^{2} M_{0} \sin k-\frac{\epsilon^{2}}{2} J k^{2} M_{2} \sin \left(k-\theta_{2}\right)-\frac{\epsilon^{2}}{8} J k^{3} M^{2} \cos k+O\left(\epsilon^{4}\right) .
\end{gathered}
$$

Evidently we need to find $M_{0}, M_{2}$, and $\theta_{2}$ in order to determine the amplitude $M$ and propagation velocity $\dot{\phi} / k$ of the pattern. The $x$-independent term in the expansion of the evolution equation gives $\dot{M}_{0}=0$, so $M_{0}$ is in fact a constant; as argued above, we can choose it to be zero, so the $M_{0}$ terms in the $M$ and $\phi$ equations can be dropped. The equations for $M_{2}$ and $\theta_{2}$ come from the coefficients of $\cos \left(2 k x-2 \phi+\theta_{2}\right)$ and $\sin \left(2 k x-2 \phi+\theta_{2}\right)$ in the evolution equation. These are best written in the form

$$
\frac{d}{d t} M_{2} e^{-i \theta_{2}}=\left(\sigma_{2 k}+i \omega_{2 k}-2 i \omega_{k}\right) M_{2} e^{-i \theta_{2}}-J k^{2} M^{2} e^{2 i k}+O\left(\epsilon^{2}\right) .
$$

Similarly, we find equations for $M_{3}$ and $\theta_{3}$,

$$
\frac{d}{d t} M_{3} e^{-i \theta_{3}}=\left(\sigma_{3 k}+i \omega_{3 k}-3 i \omega_{k}\right) M_{3} e^{-i \theta_{3}}-\frac{9}{2} J k^{2} M M_{2} e^{3 i k-i \theta_{2}}-\frac{9}{8} i J k^{3} M^{3} e^{3 i k}+O\left(\epsilon^{2}\right) .
$$


Note that in the equation for $\dot{M}(t)$, all the terms on the right are of order $\epsilon^{2}$. Therefore $M(t)$ changes on a long time scale of order $\epsilon^{-2}$, while $M_{2}$ and $\theta_{2}$ vary on times of order unity. Thus we may regard $M$ as a constant in the equations for $M_{2}$ and $M_{3}$. Since $\sigma_{2 k}$ is negative, $M_{2} \exp \left(-i \theta_{2}\right)$ goes to a quasi-steady state value which is proportional to $M^{2}$. Substituting this value into the $M$ equation gives

$$
\dot{M}=\sigma_{k} M-\epsilon^{2} \lambda M^{3}+O\left(\epsilon^{4}\right)
$$

The analytical expression for the Landau constant $\lambda$ is complicated and unenlightening; substituting (18) and (19) into it gives

$$
\lambda=16.905+64.680 \epsilon q+O\left(\epsilon^{2}\right)
$$

Note that the correction terms in the evolution equation for $M$, which would come from including higher-order terms in the original expansions (24) for the evolution equation and (31) for $h(x, t)$, are of order $\epsilon^{4}$, not $\epsilon^{3}$. As a result, the $\epsilon^{3}$ terms in the equation come only from expanding the analytical expressions for $\sigma$ and $\lambda$ in powers of $\epsilon$. This also holds for the equations for the higher harmonics. Thus we get the first-order corrections to all of our results essentially for free.

From (37) and the third-order expansion (20) for $\sigma_{k}$ we obtain the steadystate amplitude $M$ to first order in $\epsilon$,

$$
M^{2}=\left(0.25946-0.59719 q^{2}\right)-\left(0.87725-2.10774 q^{2}\right) \epsilon q+O\left(\epsilon^{2}\right)
$$

We then find the phase velocity,

$v_{p h}=\omega / k=-1+4.4934 \epsilon q+\left(1.877-4.320 q^{2}\right) \epsilon^{2}-\left(3.439-10.128 q^{2}\right) \epsilon^{3} q+O\left(\epsilon^{4}\right)$,

and the group velocity,

$v_{g r}=d \omega / d k=19.1907+13.4802 \epsilon q+\left(18.241-40.984 q^{2}\right) \epsilon^{2}-\left(43.198-107.23 q^{2}\right) \epsilon^{3} q+O\left(\epsilon^{4}\right)$,

from (33). The equation (34) for $M_{2}$ and $\theta_{2}$ gives

$$
\begin{gathered}
M_{2} / M^{2}=0.908115+0.50118 \epsilon q, \\
\theta_{2} / \pi=0.22916-0.36189 \epsilon q,
\end{gathered}
$$


and from (35) we find

$$
\begin{aligned}
M_{3} / M^{3} & =1.52273+1.89385 \epsilon q, \\
\theta_{3} / \pi & =0.4309-0.6024 \epsilon q
\end{aligned}
$$

As usual, the ripple solutions we have found are not all stable; instead, those with too large a wave number deviation $q$ are linearly unstable. The calculation of the critical value of $q$ is rather intricate, so we defer it to the Appendix. The result is that the range of stable wave numbers is rather wider than usual - it extends out to $q=0.9095 q_{0}$, where $q_{0}=\left(2 / J_{c}\right)^{1 / 2}$ is the wave number deviation at which $\sigma_{k}$ vanishes to leading order in $\epsilon$. At the edge of the stable range, the amplitude $M$ of the ripple solution is 0.4157 times its value ay $q=0$.

If instead of the NO saltation relation we use the symmetric relation (8), the results of the analysis are rather different. The expansion of $d \xi / d x$ is not as simple and clean as the derivation above; the evolution equation (24) is replaced by

$$
\begin{aligned}
& \frac{\partial h(x, t)}{\partial t}=h^{\prime \prime}(x, t)-J[h(x-1, t)-h(x, t)]^{\prime}+J\left\{[h(x-1, t)-h(x, t)] h^{\prime}(x-1, t)\right\}^{\prime} \\
& \frac{J}{2}\left\{[h(x-1, t)-h(x, t)]^{2} h^{\prime \prime}(x-1, t)\right\}^{\prime}-J\left\{[h(x-1, t)-h(x, t)]\left[h^{\prime}(x-1, t)\right]^{2}\right\}^{\prime}+\cdots
\end{aligned}
$$

We again substitute the ansatz (31) into this equation and work out the Fourier components of the result. The equations for $M$ and $\phi$ become

$$
\begin{gathered}
\dot{M}=\sigma_{k} M-\epsilon^{2} J k^{2} M_{2} M\left[\cos k \cos \theta_{2}-\cos \left(2 k-\theta_{2}\right)\right] \\
+\frac{\epsilon^{2}}{4} J k^{3} M^{3}(\sin k-2 \sin 2 k), \\
\dot{\phi}=\omega_{k}+\epsilon^{2} J k^{2} M_{2}\left[\cos k \sin \theta_{2}+\sin \left(2 k-\theta_{2}\right)\right]-\frac{\epsilon^{2}}{2} J k^{3} M^{2}(\cos k-\cos 2 k)
\end{gathered}
$$

where now $\omega_{k}$ is given by $-J k(1-\cos k)$ as is appropriate for this model. Note that the $M_{0}$ terms which were present in (32) and (33) are absent here; this is because the new saltation relation respects the symmetry under addition of a constant to $h$. The evolution of $M_{2}$ and $\theta_{2}$ is now given by

$$
\frac{d}{d t} M_{2} e^{-i \theta_{2}}=\left(\sigma_{2 k}+i \omega_{2 k}-2 i \omega_{k}\right) M_{2} e^{-i \theta_{2}}+J k^{2}\left(e^{i k}-e^{2 i k}\right) M^{2}+O\left(\epsilon^{2}\right),
$$


and the third harmonic by

$$
\begin{aligned}
\frac{d}{d t} M_{3} e^{-i \theta_{3}}=\left(\sigma_{3 k}\right. & \left.+i \omega_{3 k}-3 i \omega_{k}\right) M_{3} e^{-i \theta_{3}}+\frac{3}{2} J k^{2} e^{i k}\left(1-e^{i k}\right)\left(1+3 e^{i k}\right) M M_{2} e^{-i \theta_{2}} \\
& +\frac{3}{8} i J k^{3} e^{i k}\left(1-e^{i k}\right)\left(1-3 e^{i k}\right) M^{3}+O\left(\epsilon^{2}\right)
\end{aligned}
$$

After carrying out the calculation we find a much larger value for the Landau coefficient,

$$
\lambda=151.26+88.014 \epsilon q+O\left(\epsilon^{2}\right) .
$$

Thus for a given wave number, the restabilized amplitude $M$ of the ripples is smaller by a factor of about 3 :

$$
M^{2}=\left(0.0289975-0.066743 q^{2}\right)-\left(0.003966-0.0190315 q^{2}\right) \epsilon q+O\left(\epsilon^{2}\right) .
$$

The phase velocity is more negative than before, as we found from the linear stability analysis,

$v_{p h}=-5.6033+4.4934 \epsilon q-\left(1.506-1.16475 q^{2}\right) \epsilon^{2}-\left(0.197-1.853 q^{2}\right) \epsilon^{3} q+O\left(\epsilon^{4}\right)$,

and likewise the group velocity,

$v_{g r}=14.5874+13.4802 \epsilon q-\left(2.569-4.612 q^{2}\right) \epsilon^{2}-\left(8.152-19.799 q^{2}\right) \epsilon^{3} q+O\left(\epsilon^{4}\right)$.

For a given amplitude, however, the harmonics are stronger than before: we find

$$
\begin{aligned}
M_{2} / M^{2} & =1.41691+0.213856 \epsilon q, \\
\theta_{2} / \pi & =x 0.4443-0.2027 \epsilon q,
\end{aligned}
$$

and

$$
\begin{gathered}
M_{3} / M^{3}=2.89266+1.57014 \epsilon q, \\
\theta_{3} / \pi=-0.1426-0.3952 \epsilon q .
\end{gathered}
$$

The range of wave numbers for which these solutions is stable is somewhat narrower than before but still wider than usual, extending out to $q=0.7571 q_{0}$, where the amplitude $M$ is 0.6533 times its value at $q=0$. 


\section{Numerical solutions}

We now present numerical solutions and compare them with the predictions made in the previous section. The nonlocal evolution equation (7) was solved numerically with periodic boundary conditions on a system of length $l=2 \pi / k$, so that only the Fourier modes $n k$ contributed to the solutions. For the discretization scheme, we chose an explicit method using forward differences in time and central differences in space. The axis was discretized at $2^{9}$ equally spaced sites with $\Delta x=2 \pi / k \bullet 2^{-9}$ and solutions were generated for five different values of $J$ near $J_{c}=4.603$, namely $J=4.62,4.65,4.70,4.75,4.80$, and values of $k$ were chosen to span the unstable region. Initial conditions were sinusoids of wave number $k$ centered around $h=0$. At $\mathrm{t}=0$, we start with a sinusoid at a particular wave number $k$, and let it evolve with $(\Delta x)^{2} / \Delta t=1 / 4$ until it reaches a steady state. This takes about $10^{6}$ time steps. The nonlocal term in the evolution equation, $J(d \xi / d x-1)$, was evaluated for a given $x$ by finding the nearest upwind value of $\xi$ satisfying the equation $x=\xi+1+h(\xi)$. Spefifically, the first root of the function $f(\xi ; x)=\xi+1+h(\xi)-x$ with value less than $x$ was obtained by simply finding the two sites upwind of $x$ and nearest to it between which $f(\xi ; x)$ changed sign. Then, $d \xi / d x$ was calculated using the values of $h$ at these sites. The final steady state, $h(x, t)$, is then Fourier transformed, i.e.:

$$
h(x, t)=\sum_{n=1}^{\infty}\left[a_{n}(t) \sin n k x+b_{n}(t) \cos n k x\right]
$$

from which we obtain $M_{n}=\left(a_{n}^{2}+b_{n}^{2}\right)^{1 / 2}$ and $\theta_{n}=\tan ^{-1}\left(b_{n} / a_{n}\right)$. Note that $M_{1}=M$ and $\theta_{1}=\phi$ in this notation. The nonlinear analysis in the previous section predicts that these quantities will go to time-independent values. We find numerically that they actually oscillate as a function of time around their mean values. However, the magnitudes of these oscillations are quite small and decrease with increasing grid resolution, so we believe them to be numerical artifacts. We therefore take the time averaged mean values and compare them with the predictions of the weakly nonlinear analysis.

We also find that although we start with an initial profile with the average height $h_{0}=0$, the mean position of the steady state pattern shifts slightly upward in some cases, downward in others, to a small but finite $h_{0}$. Since the mean height of the sandbed is conserved by the exact evolution equation, 
we believe that this is also a numerical artifact. Moreover, as mentioned in section 2, we can map any steady state solution with finite $h_{0}$ to the solution with $h_{0}=0$ by redefining the horizontal length scale from $L_{0}$ to $L_{0}\left(1+h_{0}\right)$ and shifting the control parameter from $J$ to $J\left(1+h_{0}\right)$. However, the magnitude offset $h_{0}$ was always of the order of $10^{-5}$ to $10^{-3}$, and thus in all cases studied here, the corrections due to such an offset are quite negligible. Hence, our results without these corrections are virtually identical to those with corrections.

Figure 2 shows the amplitude $M$ of the fundamental mode as a function of $k$ for different values of $J$. The data points are fairly close to the values predicted by the first-order expansion (38), which are represented by continuous curves. Note that the curves are asymmetric around the critical value $k_{c}$ and the asymmetry becomes more pronounced for larger $J$. The weakly nonlinear analysis is capable of predicting this asymmetry only because the order- $\epsilon$ terms are included.

In Figure 3 we plot the phase velocity of the fundamental mode against $k$ for different values of $J$. The speed was obtained by calculating $\phi(t)$ in the expression $\cos (k x-\phi(t))$, which is proportional to the fundamental mode in the steady state. The function $\phi(t)$ was found to be linear in $t$, so $v$ was calculated as $v=d \phi(t) / d t / k$. The data points are compared against the weakly nonlinear predictions (solid line) given by (39). Only for fairly large $J$, and only near the high-wave-number end of the band of ripple solutions, does the velocity become positive, that is, in the direction of the wind.

In Figures $4 \mathrm{a}$ and $4 \mathrm{~b}$ are plotted the ratios of the amplitudes of the second and third harmonics to the appropriate powers of the fundamental ampltude, i.e., $R_{2}=M_{2} / M^{2}$ and $R_{3}=M_{3} / M^{3}$. The data fit quite well with the theoretical predictions for both cases, in particular near the onset $k_{c}=4.493$, where the nonlinear analysis is most reliable. Note that the first-order terms in the analytical results match the slope of the numerical results. The curvature which is evident in the numerical data for $k$ farther from $k_{c}$ is apparently a higher-order effect. Note that the width of the band of ripple solutions increases with $\epsilon$, so an appreciably large $\epsilon$ is required to reach these larger values of $\left|k-k_{c}\right|$.

In Figures $5 \mathrm{a}$ and $5 \mathrm{~b}$ we plot the phase angles $\theta_{2}$ and $\theta_{3}$ against $k$. The agreement between the simulations and the weakly nonlinear analysis is again quite strong for $J$ near onset. The order- $\epsilon$ terms in the analytical results match the slope of the numerical data. For higher $J$ we observe a systematic 
downward deviation in the numerical results. The shift appears to be linear in $J$, and so is second-order in $\epsilon \equiv\left(J-J_{c}\right)^{1 / 2}$.

\section{Discussion}

We have carried out numerical and weakly nonlinear analyses of the NishimoriOuchi continuum model [3,11] for windblown sand, and also for a modification of that model which respects the physical symmetry of the system under changes of the reference level of height. Both versions of the model yield the surprising result that the ripple patterns, which form when the flat sandbed becomes unstable, drift upwind even as the sand which forms the ripples is blown downwind. This drift is found in the linear stability analysis and persists in the weakly nonlinear results, and numerical integrations confirm that it is a real consequence of the model. Such a counterintuitive result has not been examined or detected by previous Monte Carlo simulations of this model $[3,11]$ or in real experiments [10]. It would be interesting to check experimentally whether or not ripples can move against the wind. The symmetric version of the model actually predicts a considerably higher upwind drift speed than the original Nishimori-Ouchi version.

It may also be surprising that the differences between the symmetric and Nishimori-Ouchi models are merely qualitative. The restabilized ripple pattern for a given value of the control parameter have smaller amplitudes (by a factor of about 3) and higher drift velocities (by a factor of over 5) in the symmetric model than in the original version. The relative sizes and phases of the higher harmonics in the ripple shape are also different for the two models.

A number of modifications to the model are needed in order to make it comparable with experiments. A major ingredient that is left out of the model is any effect of the surface topography on the wind. This lack means that there is no shadowing effect in the model. Including such an effect would make it more likely for grains to settle on the downwind side of a ripple than on the upwind side, and more likely for them to be blown off the upwind side than the downwind side. This would likely reduce the tendency of the ripples to drift upwind. The result that the ripples drift upwind in this model, which neglects shadowing, may be an indirect indication of the importance of shadowing in the development of real ripple patterns. An improved model 
of creep may also be needed; a downwind bias in the creep would modify the drift velocity. Perhaps most critical is a better and more realistic form of the saltation function, which must account the effects of the topography of the sandbed, and the many particle dynamics of the grains in the air as well as on the surface. 


\section{References}

[1] R.A. Bagnold, Proc. Roy. Soc. A157, 594 (1936); See also The physics of blown sand and desert dunes, reprinted by Chapman and Hall (1981).

[2] K. Pye and H. Tsoar, Aeolian sand and sand dunes, Unwin Hyman, London (1990).

[3] H. Nishimori and N. Ouchi, Phys. Rev. Lett. 71, 197 (1993).

[4] R.S. Anderson, Sedimentology, 34, 943 (1987).

[5] O. Terzidis, P. Claudin, and J.P. Bouchaud, cond-matt/9801295.

[6] For recent observations of surface instabilities that develop when grains are subject to vibrations, see: F. Melo, P. Umbanhowar, and H.L. Swinney, Phys. Rev. Lett. 75, 3838 (1995); T.H. Metcalf, J.B. Knight, and H.M. Jaeger, Physica A 236, 202 (1997); K.M. Aoki and T. Akiyama, Phys. Rev. Lett. 77, 4166 (1996).

[7] See, e.g.: J.S. Langer, Science 243, 1150 (1989); D.A. Kessler, H. Levine, and J. Koplik, Adv. Phys. 37, 255 (1988); Dynamics of curved fronts, edited by P. Pelce, Academic, San Diego, (1988) and references therein.

[8] See, e.g., M. Ben-Amar and B. Moussallam, Phys. Rev. Lett. 60, 317 (1989) and references therein.

[9] D. A. Kurtze and D. C. Hong, J. Korean. Phys. Soc. 28(2), 178 (1995) and references therein.

[10] A. Betat, V. Frette, and I. Rehberg, Phys. Rev. Lett. 83, 88 (1999).

[11] More detailed description of the model can be found in: H. Nishimori and N. Ouchi, Int. J. Mod. Phys. B. Vol.7, \# 9 \& 10, 2025 (1995). 


\section{A Stability of ripple solutions and the Eck- haus boundary}

In this section, we examine the stability of the ripple solutions, and so determine the Eckhaus boundary in the $J-k$ plane, within which the solutions are linearly stable and so may be observed. We begin with the ripple solution

$$
h_{0}(x, t)=\epsilon M \cos (k x-\omega t)+\epsilon^{2} M_{2} \cos \left[2(k x-\omega t)+\theta_{2}\right]+O\left(\epsilon^{3} M_{3}\right),(A-1)
$$

with $k=k_{c}+\epsilon q$, and add an infinitesimal perturbation $h_{1}(x, t)$. If the perturbation contains a Fourier component with wave number $k+\epsilon q^{\prime}$, then the nonlinear terms in the evolution equation will generate a component with wave number $k-\epsilon q^{\prime}$. Thus we will start the calculation by taking $h_{1}$ to have the form

$h_{1}(x, t)=A_{-}(t) \cos \left[\left(k-\epsilon q^{\prime}\right) x-\left(\omega t+\phi_{-}(t)\right)\right]+A_{+}(t) \cos \left[\left(k+\epsilon q^{\prime}\right) x-\left(\omega t+\phi_{+}(t)\right)\right]$.

Substituting this into the evolution equation, expanding, and picking off the coefficients of the sines and cosines of $\left(k-\epsilon q^{\prime}\right) x$ and $\left(k+\epsilon q^{\prime}\right) x$ yields a closed set of equations for the amplitudes $A_{-}$and $A_{+}$and the phase $\phi_{+}+\phi_{-}$. These equations have the form

$$
\begin{gathered}
\dot{A}_{-}=\Sigma_{-} A_{-}+\alpha A_{+} \cos \psi \\
\dot{A}_{+}=\Sigma_{+} A_{+}+\alpha A_{-} \cos \psi \\
\dot{\psi}=\Omega-\alpha\left[\left(A_{+} / A_{-}\right)+\left(A_{-} / A_{+}\right)\right] \sin \psi
\end{gathered}
$$

where $\psi$ is $\phi_{+}+\phi_{-}$plus a constant which depends on $k$ and $q^{\prime}$ (but not time), and the overdot denotes a derivative with respect to the slow time variable $\epsilon^{2} t$. The coefficients are given to leading order in $\epsilon$ by

$$
\begin{gathered}
\Sigma_{ \pm}=\frac{k_{c}^{2}}{2}\left[\left(\frac{k_{c}^{2}}{2}-\frac{2 \lambda}{k_{c}^{2}}\right) M^{2} \mp 2 q q^{\prime}-q^{\prime 2}\right] \\
\alpha=\alpha_{0} k_{c}^{2} M^{2}=1.98183 k_{c}^{2} M^{2} \\
\Omega=\frac{1}{2} k_{c}^{3} M^{2}+3 k_{c} q^{\prime 2}
\end{gathered}
$$


Note that it is important to keep the second-order term in $h_{0}$ during the calculation, since it contributes to $\alpha$. (Omitting it changes $\alpha_{0}$ to 2.58559, a $30 \%$ change.)

We must now determine whether the amplitudes given by (A-3) grow or decay with time. We can simplify the equations somewhat by defining

$$
R=A_{+} / A_{-}
$$

the amplitude equations then become

$$
\begin{gathered}
\dot{A}_{-}=\left(\Sigma_{-}+\alpha R \cos \psi\right) A_{+}, \\
\dot{R}=\left(\Sigma_{+}-\Sigma_{-}\right) R+\alpha\left(1-R^{2}\right) \cos \psi, \\
\dot{\psi}=\Omega-\alpha\left[\left(1+R^{2}\right) / R\right] \sin \psi .
\end{gathered}
$$

Note that the first equation decouples from the last two. If it happens that $R$ and $\psi$ go to constants as $t \rightarrow \infty$, then the amplitudes decay for $\Sigma_{-}+$ $\alpha R \cos \psi<0$ and grow otherwise. Thus for a given $q$, the ripple state (A1 ) is linearly stable if this inequality is satisfied for every $q^{\prime}$, otherwise it is unstable. To see what $R$ and $\psi$ actually do, we combine the $R$ and $\psi$ equations into an evolution equation for the complex variable

$$
Z=R \exp (i \psi),
$$

namely

$$
\dot{Z}=\alpha\left(1-Z^{2}\right)+\left(\Sigma_{+}-\Sigma_{-}+i \Omega\right) Z .
$$

Clearly this equation has two fixed points, and solving it exactly reveals that the one with positive real part is a global attractor and the one with negative real part a global repeller. Thus $R \cos \psi$ does go to a constant, and from its value we can decide whether the ripple state is stable or not.

Since it is the real part of $Z$, namely $R \cos \psi$, which determines whether the perturbation grows or decays, it is useful to rewrite (A-8) in terms of the real and imaginary parts of $Z$,

$$
Z=X+i Y \text {. }
$$

We find

$$
\dot{X}=\alpha\left(1-X^{2}\right)+\left(\Sigma_{+}-\Sigma_{-}\right) X-\Omega Y+\alpha Y^{2},
$$




$$
\dot{Y}=\left(\Sigma_{+}-\Sigma_{-}\right) Y+\Omega X-2 \alpha X Y .
$$

By linearizing about a fixed point $(X, Y)$ of this system, we quickly find that the fixed point is an attractor for $X>\left(\Sigma_{+}-\Sigma_{-}\right) / 2 \alpha$. To find the fixed points, we set $\dot{X}=\dot{Y}=0$ and solve the second equation for $Y$ in terms of $X$, then substitute into the first equation to get

$$
\alpha-X\left(\alpha X-\Sigma_{+}+\Sigma_{-}\right)=\frac{\Omega^{2} X\left(\alpha X-\Sigma_{+}+\Sigma_{-}\right)}{\left(2 \alpha X-\Sigma_{+}+\Sigma_{-}\right)^{2}} .
$$

The two sides of this equation are plotted in Fig. 6. Both sides are symmetric about $X=\left(\Sigma_{+}-\Sigma_{-}\right) / 2 \alpha$, so there is clearly one solution with $X$ greater than this - the attractor - and one, the repeller, with $X$ less. In order for the perturbation to decay, the attractor must have $X$ less than $-\Sigma_{-} / \alpha$. From the plot, we see that this means that at $X=-\Sigma_{-} / \alpha$ the right side of (A-11) must be greater than the left side. After a little algebra, we can write this condition for the perturbation to decay in the form

$$
\Sigma_{+} \Sigma_{-}>\frac{\alpha^{2}\left(\Sigma_{+}+\Sigma_{-}\right)^{2}}{\left(\Sigma_{+}+\Sigma_{-}\right)^{2}+\Omega^{2}}
$$

Equation (A-12) above is the condition for the amplitude of a perturbation with a specific value of $q^{\prime}$ to decay. In order to conclude that the ripple solution with a given $q$ is linearly stable, we must see to it that this condition is satisfied for all $q^{\prime}$. For this we must substitute for the parameters from (A-4) above. To put the result into a useful form, we define $Q=2 q^{\prime 2} / k_{c}^{2} M^{2}$ and eliminate $q^{2}$ in favor of $M^{2}$. After some rearranging, we find that the condition for the solution to be stable is

$$
M^{2}>\frac{16 Q}{J} \frac{k_{c}^{2}(\beta+Q)^{2}+(1+3 Q)^{2}}{k_{c}^{2}\left[(\beta-Q)^{2}+4 Q\right]\left[k_{c}^{2}(\beta+Q)^{2}+(1+3 Q)^{2}\right]-16 \alpha_{0}^{2}(\beta+Q)^{2}},
$$

where $\beta=1-\left(4 \lambda / k_{c}^{2}\right)=0.834132$. The complicated function of $Q$ on the right has a single maximum for positive $Q$, at a height of 0.04484 . Ripple states with $M^{2}$ below this are unstable, while those with $M^{2}$ larger than this are linearly stable. From this we find that the range of wave numbers of linearly stable ripple solutions is given by $|q|<0.9095 q_{0}$, where $q_{0}=\left(2 / J_{c}\right)^{1 / 2}$ is the largest wave number for which a ripple solution exists. 
In summary, we have found that in the weakly nonlinear regime, the flat sandbed is unstable against perturbations with wave numbers $k$ in the range

$$
\left|k-k_{c}\right|<\epsilon q_{0}=\sqrt{2\left(\frac{J}{J_{c}}-1\right)},
$$

while the Eckahus boundary is given by

$$
\left|k-k_{c}\right|<0.9095 \epsilon q_{0}=\sqrt{1.654\left(\frac{J}{J_{c}}-1\right)} .
$$

For the symmetric saltation relation, the structure of the calculation is the same but the numbers are different. We find that the marginally stable wave number is given by $q=0.7571 q_{0}$, so the Eckhaus boundary is now given by

$$
\left|k-k_{c}\right|<0.7571 \epsilon q_{0}=\sqrt{1.1465\left(\frac{J}{J_{c}}-1\right)} . \quad(A-16)
$$




\section{Figure Captions}

Fig. 1: Saltation refers to the process of a single grain being ejected from the surface at a point $\xi$ and being blown to a landing point $x$ by the wind.

Fig. 2: The steady state amplitude $\epsilon M$ vs. $k$ for five different values of $J$. The continuous lines are the analytical predictions given by Eq. (38).

Fig. 3: The propagation speed of the steady state patterns $v_{p h}$ vs. $k$ for different values of $J$. Numerically obtained values are compared to the analytical predictions (continuous curves) from Eq. (39).

Fig. 4: The ratios (a) $R_{2}=M_{2} / M^{2}$ and (b) $R_{3}=M_{3} / M^{3}$ are plotted against $k$. The solid line is the analytical predictions from Equations (41) and (42). Fig. 5: (a) $\theta_{2}$ and (b) $\theta_{3}$ are plotted against $k$ for five different values of $J$. The solid line is the analytical predictions from Equations (41) and (42).

Fig. 6: The right and left hand sides of Eq. (A-11). 


\section{Typical Saltation Trajectory and Related Variables}

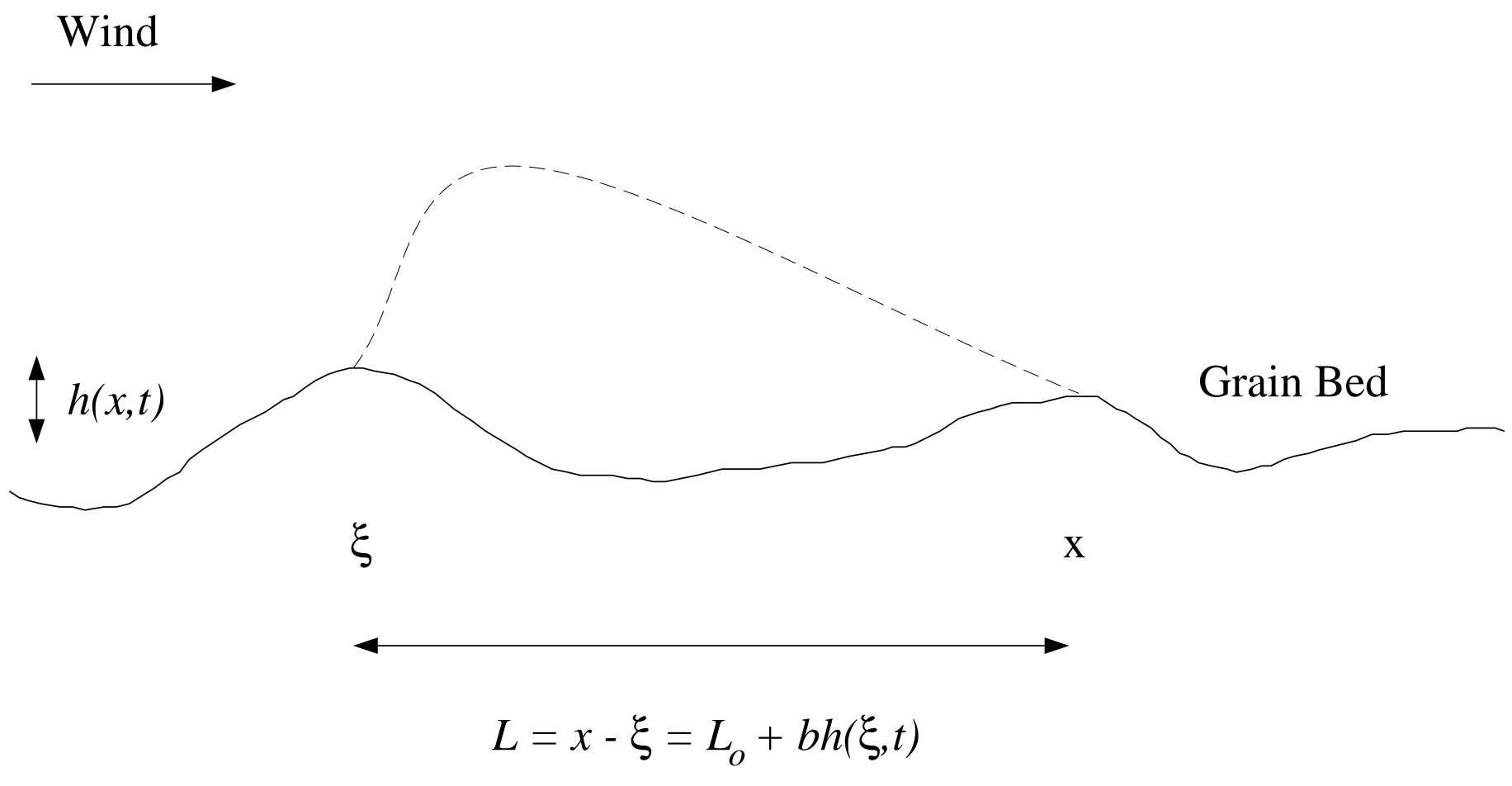




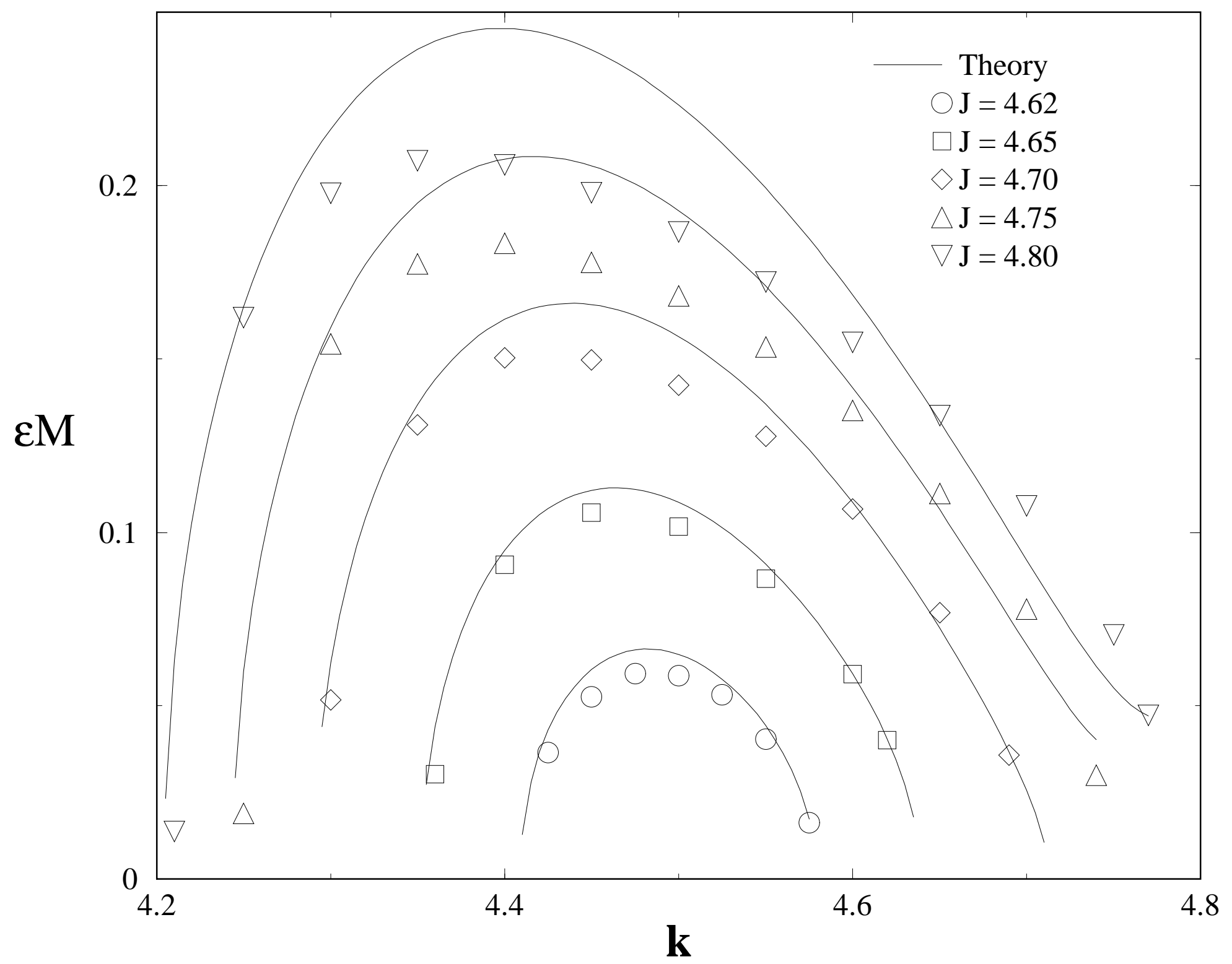




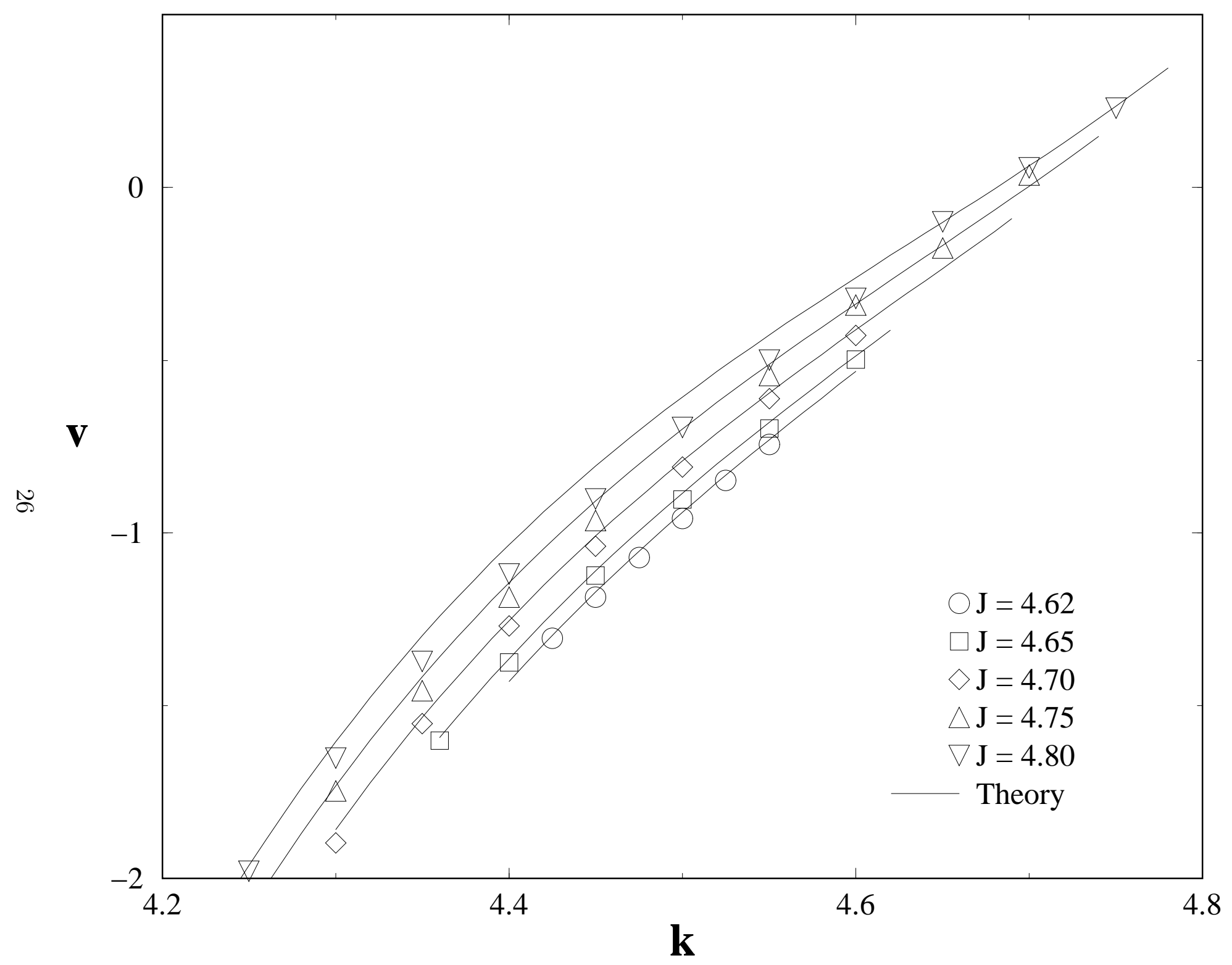




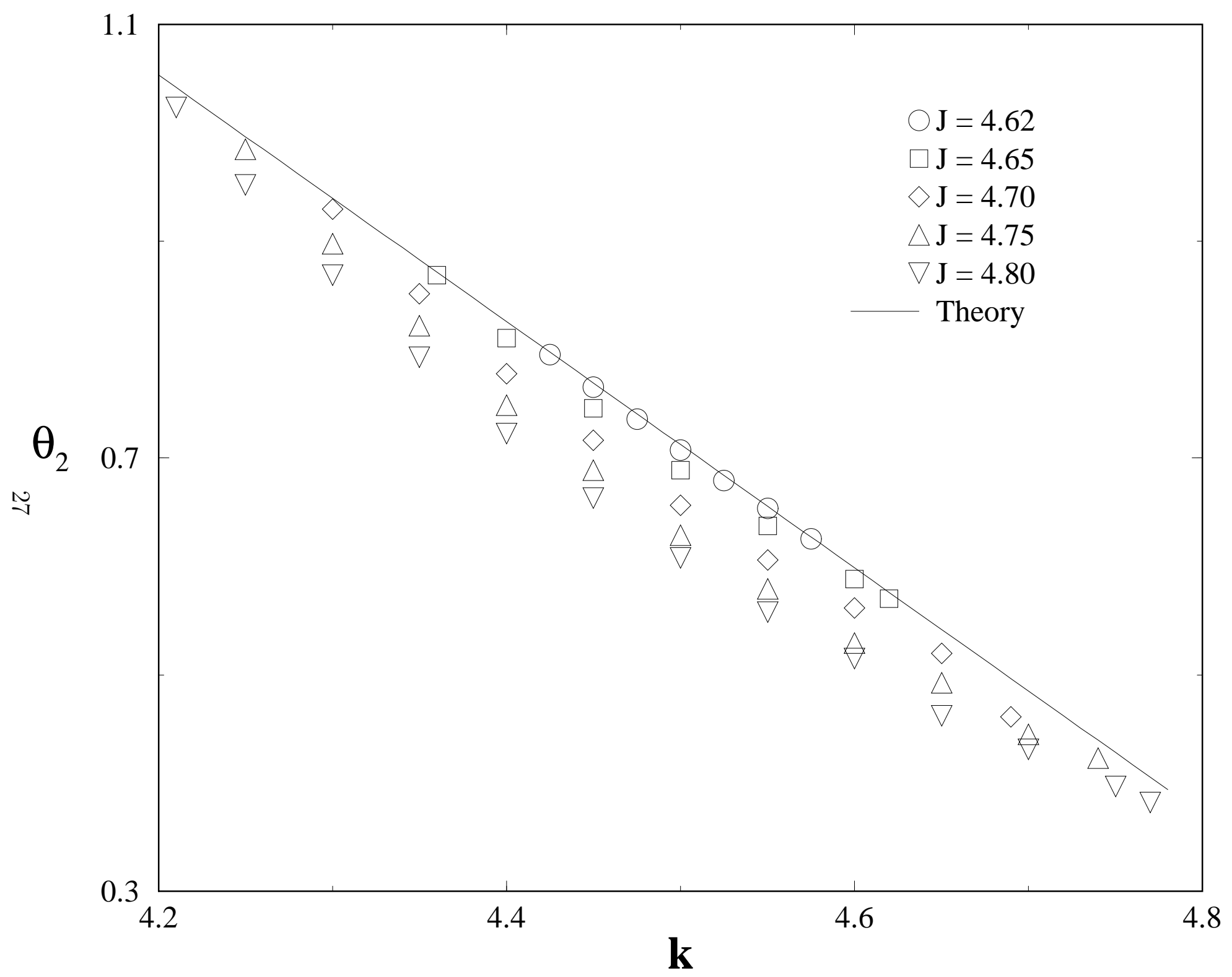




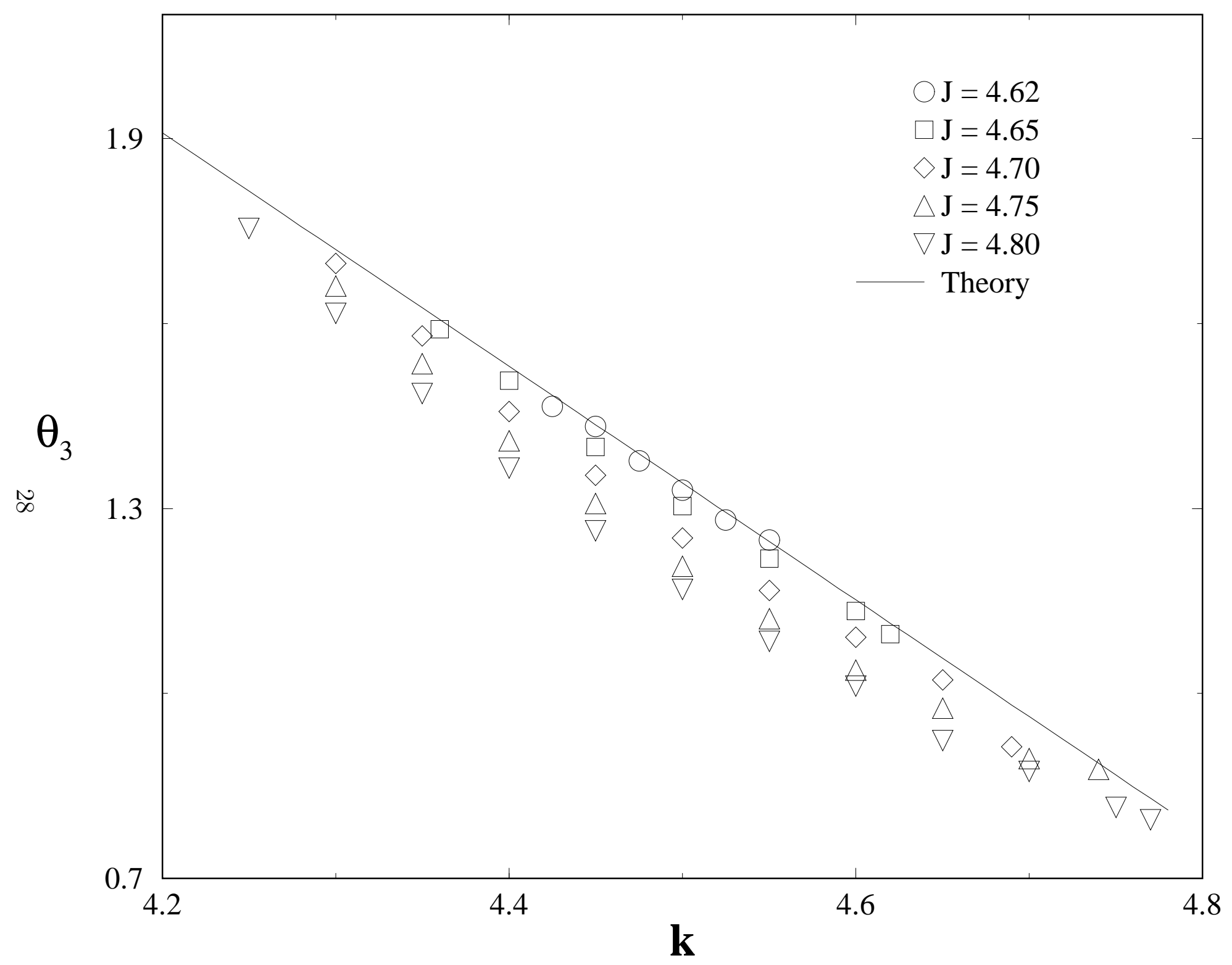




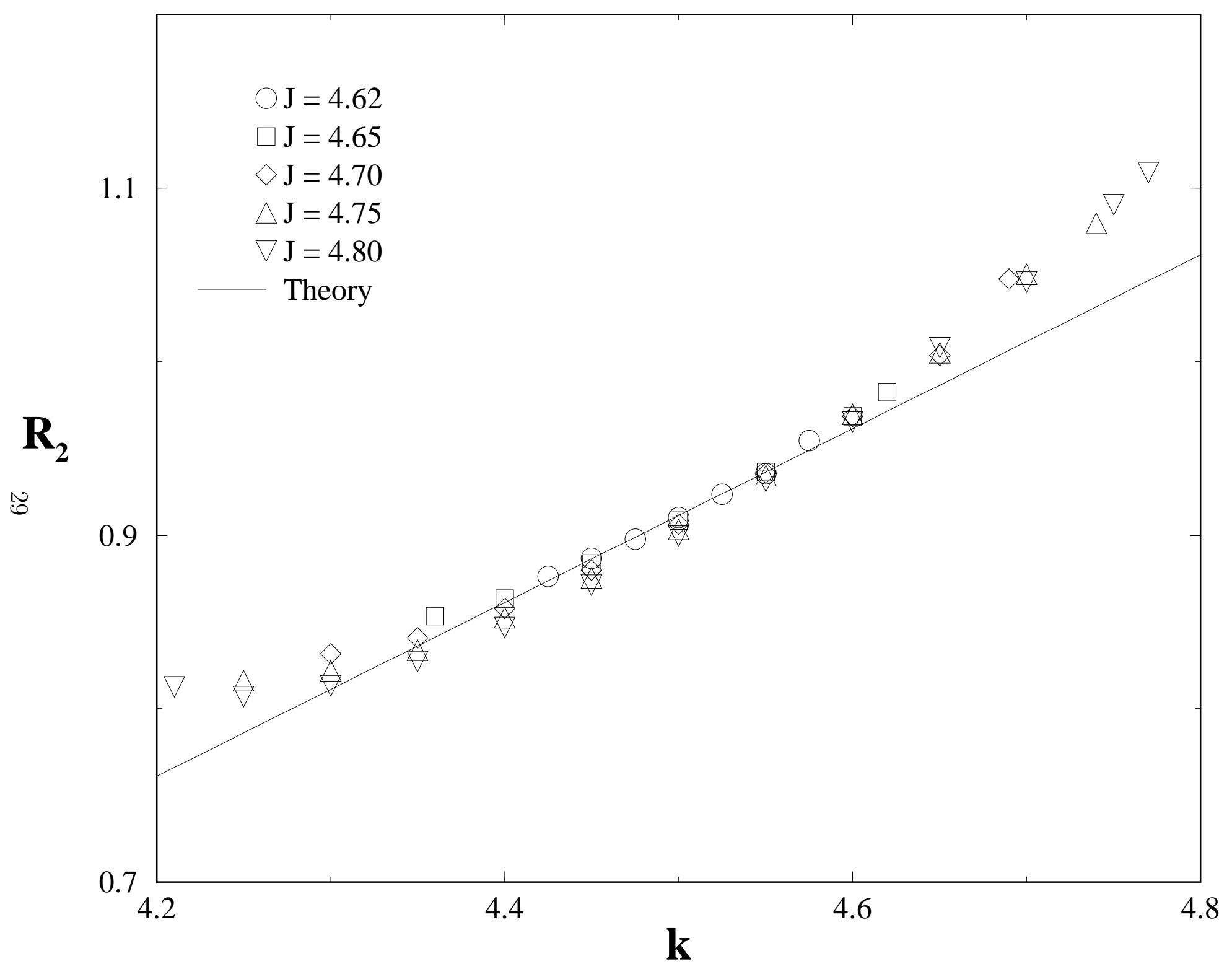




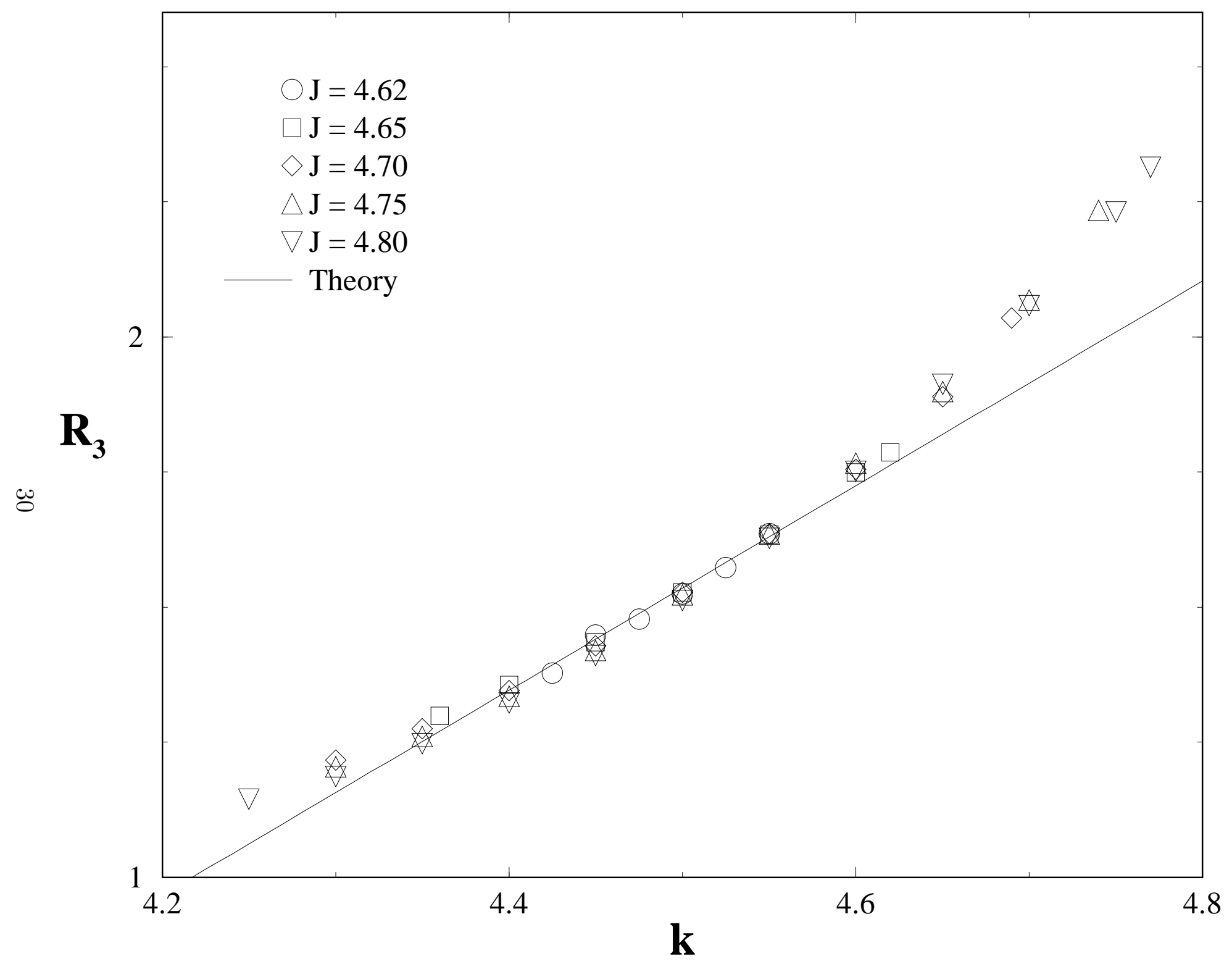




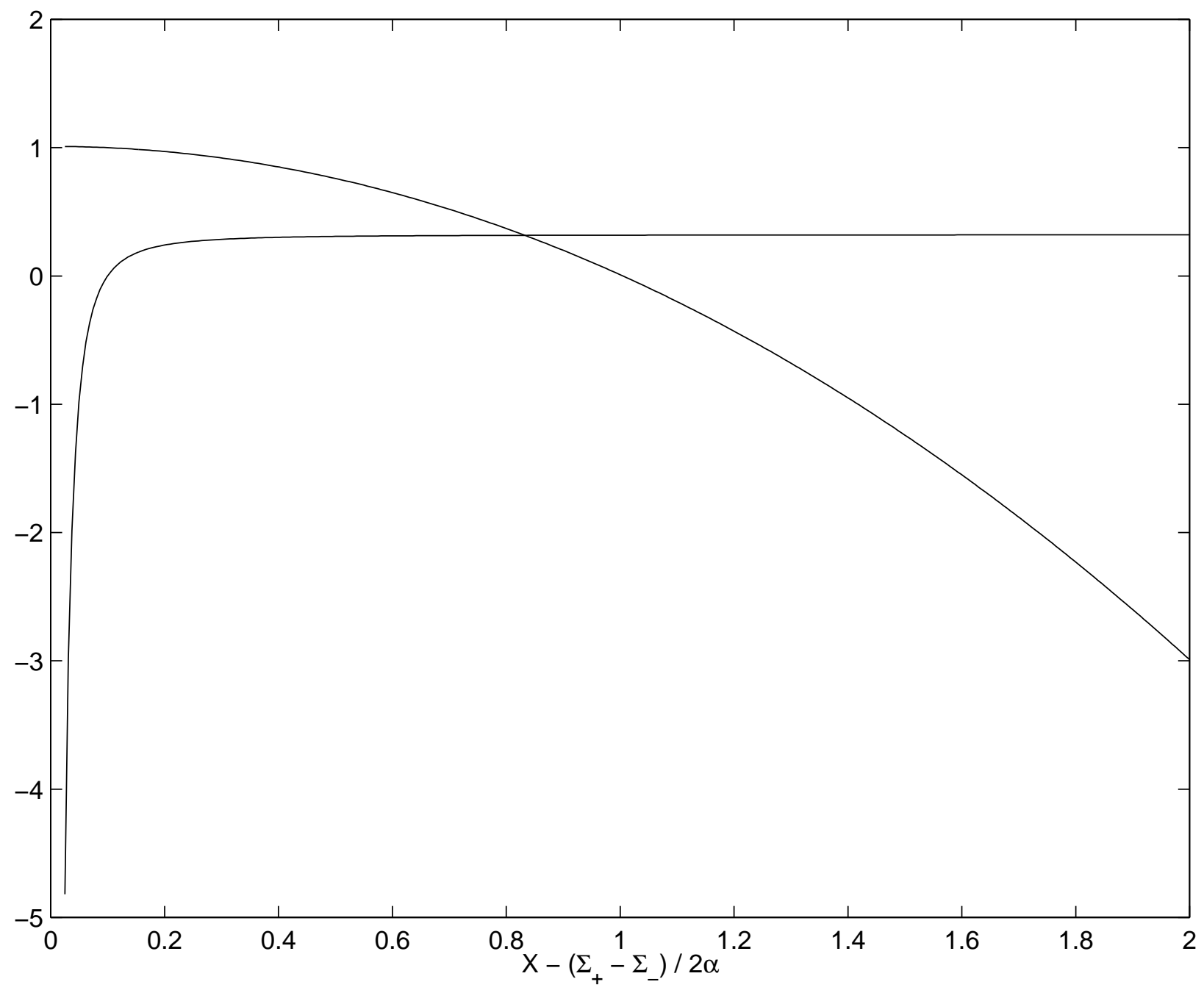

